\title{
Communication \\ Periprocedural outcomes of protamine administration after catheter ablation of atrial fibrillation
}

\author{
Jakrin Kewcharoen ${ }^{1}$, Kuldeep Shah ${ }^{2}$, Rahul Bhardwaj ${ }^{1}$, Tahmeed Contractor ${ }^{1}$, \\ Mohit K. Turagam ${ }^{3}$, Ravi Mandapati ${ }^{1}$, Dhanunjaya Lakkireddy ${ }^{4}$, Jalaj Garg ${ }^{1, *}$ \\ ${ }^{1}$ Division of Cardiology, Cardiac Arrhythmia Service, Loma Linda University Health, Loma Linda, CA 92354, USA \\ ${ }^{2}$ Department of Cardiovascular Medicine, Beaumont Hospital, Oakland University William Beaumont School of Medicine, Royal Oak, MI 48309, USA \\ ${ }^{3}$ Helmsley Electrophysiology Center, Icahn School of Medicine at Mount Sinai, New York, NY 10029, USA \\ ${ }^{4}$ Kansas City Heart Rhythm Institute and Research Foundation, Kansas City, KS 66211, USA \\ *Correspondence: garg.jalaj@yahoo.com (Jalaj Garg) \\ Academic Editor: Robert C. Hendel \\ Submitted: 2 November 2021 Revised: 8 December 2021 Accepted: 8 December 2021 Published: 19 January 2022
}

\begin{abstract}
Background: Perioperative anticoagulation management with uninterrupted or minimally interrupted anticoagulation during atrial fibrillation $(\mathrm{AF})$ ablation is thought to be critical to minimize thromboembolic complications. Protamine is often administered to neutralize the effects of heparin and expedite vascular hemostasis post-procedure. Objective: We performed a systematic review and meta-analysis to determine the effectiveness of protamine to expedite vascular hemostasis and ambulation in patients undergoing AF ablation. Methods: Electronic searches on PubMed, The Cochrane Library, EMBASE, EBSCO, Web of Science, and CINAHL databases from the inception through August 7, 2021, were performed. The primary outcomes included - time to hemostasis (minutes) and time to ambulation (minutes). The secondary outcomes included - any vascular complications (excluding minor hematoma), minor hematoma, or cerebrovascular accidents (CVA). Results: A total of 5 eligible studies ( 3 retrospective cohort studies and two randomized trials) consisting of 1012 patients (515 patients received protamine group and 497 patients did not receive protamine group) were included in the meta-analysis. There was a significant reduction in time to ambulation [weighted mean difference (WMD) -176.6 minutes, $95 \%$ Confidence interval (CI) -266.9 to $-86.3 ; p<0.01$ ] and time to hemostasis (WMD -13.72 minutes, $95 \% \mathrm{CI}-22$ to $-5.4, p<0.01$ ) in the protamine group compared to the contrary. At a follow-up up to 3 months, there was no statistical difference between the two groups with regards to vascular complications ( $2.9 \%$ vs. $7.4 \%$; Risk ratio (RR) $0.4695 \%$ CI 0.17 to $1.24 ; p=0.12)$, minor hematoma $(2.1 \%$ vs. $5.8 \%$; RR 0.43 , $95 \%$ CI 0.16 to $1.2 ; p=0.11$ ) or CVA ( 0 vs. $0.3 \%$; RR $0.62,95 \%$ CI 0.08 to $4.98 ; p=0.65$ ). Conclusion: Protamine administration was associated with reduced time to ambulation (176 minutes reduction) and time to hemostasis (13 minutes reduction) without an increase in any adverse events.
\end{abstract}

Keywords: Atrial fibrillation ablation; Protamine; Anticoagulation

\section{Introduction}

Therapeutic anticoagulation with unfractionated heparin (UFH) during atrial fibrillation (AF) ablation is critical to minimize procedure-related thromboembolic complications [1]. The 2017 HRS/EHRA/ECAS/APHRS/SOLAECE, an expert consensus statement on catheter ablation of $\mathrm{AF}$, recommends protamine to neutralize effects of heparin and hasten hemostasis to avoid vascular complications such as aneurysm, fistula, and hematoma (Class IIa, based on moderate quality of evidence) [1]. However, its safety profile and efficacy in promoting faster patient recovery are still unclear. We performed a systematic review and meta-analysis to determine the effectiveness of protamine to expedite vascular hemostasis and ambulation in patients undergoing AF ablation.

\section{Methods}

A systematic search on PubMed, The Cochrane Library, EMBASE was done using the keywords: "protamine" and "atrial fibrillation ablation". Two investigators (JK and KS) independently performed the literature search (using PubMed, EMBASE, SCOPUS, Google Scholar, and ClinicalTrials.gov from inception to October 20th, 2021) and screened all titles and full-text versions of all relevant studies that met study inclusion criteria (Supplementary Fig. 1).

We used the following keywords and medical subject heading: "Protamine", "atrial fibrillation ablation", "AF ablation". This meta-analysis was performed according to the PRISMA guidelines and was prospectively enrolled in the PROSPERO database (ID 288480).

The eligibility criteria for our systematic review and meta-analysis included: (1) all studies reporting outcomes of the use of protamine in patients with AF ablation (2) studies that included human subjects. We included studies only 


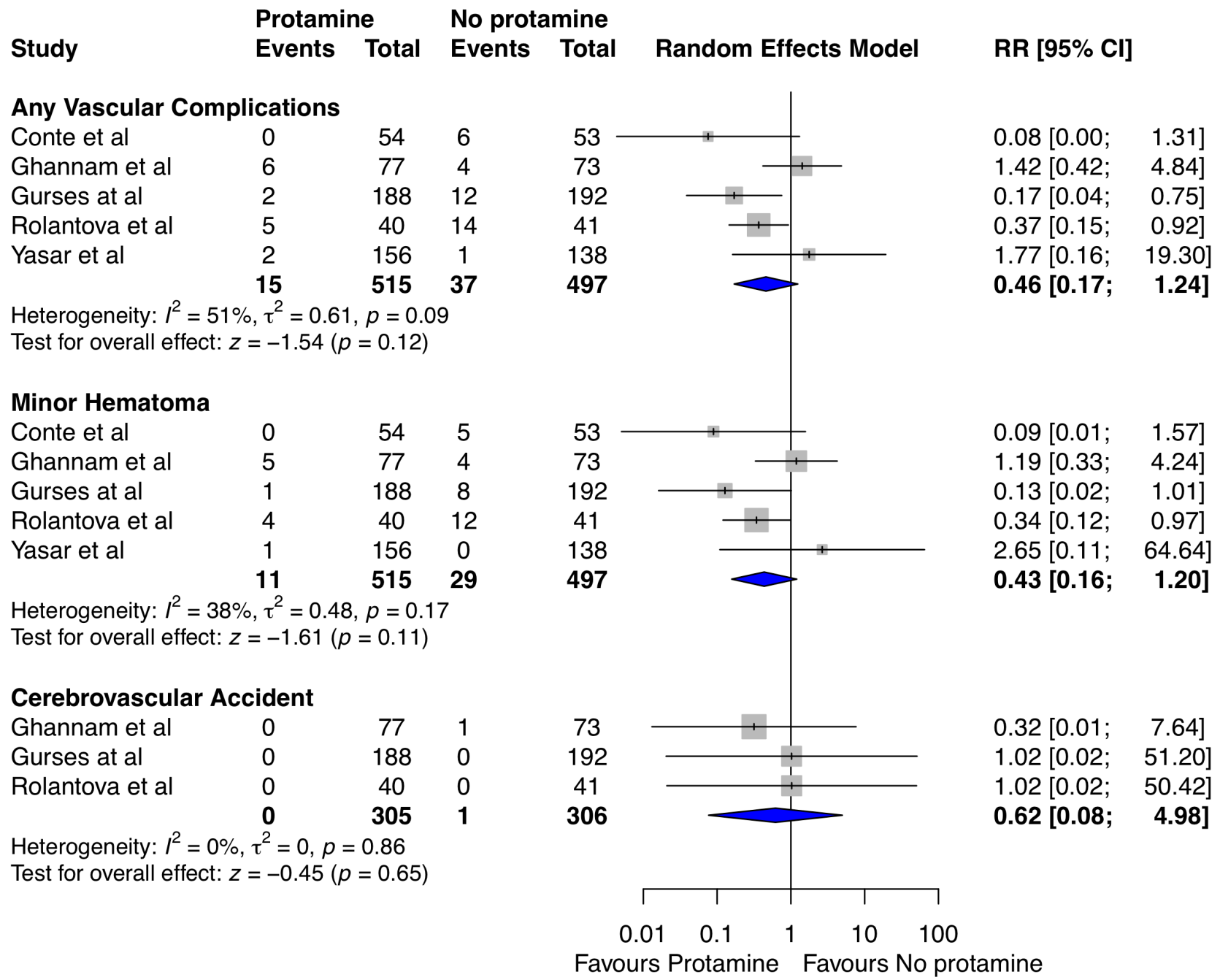

Fig. 1. Forest plot demonstrating the WMD of time to ambulate and time to hemostasis post-procedure between patients who received protamine and patients who did not. The total number of participants mean and SD for time to ambulation and time to hemostasis are reported for each study individually. Square with horizontal line represents WMD and 95\% CI for each individual study, with square size reflecting the statistical weight of the study using the random-effects model. The diamond represents pooled WMD and 95\% CI for each outcome. Heterogeneity $\left(I^{2}\right)$ and between-study variance $\left(T^{2}\right)$ with $p$-value, and overall effect size $(z)$ with $p$-value are reported below each of their respective forest plot. CI, Confidence interval; SD, Standard deviation, WMD, Weight mean difference.

in the English language. Case reports, abstracts, editorial, or systematic reviews were excluded. The data from the included studies were extracted using a standardized protocol and a data extraction form. Any discrepancies between the two investigators were resolved with a consultation with the co-senior investigators (DL and JG). The following data was extracted from the eligible studies: author name, study design, publication year, follow-up duration, number of patients, age, gender, co-morbid conditions, anticoagulation type, ablation strategy, procedural characteristics and post ablation management. The Cochrane - Risk bias assessment tool was used to appraise the quality of randomized studies (Supplementary Table 1), while the Newcastle Ottawa Risk bias assessment tool was used to appraise the quality of the included studies (Supplementary Table 2). Studies' quality was rated as good, fair, and poor by award- ing stars in each domain. A "good" quality score required 3 or 4 stars in the selection, 1 or 2 stars in comparability, and 2 or 3 stars in outcomes. A "fair" quality score required two stars in the selection, 1 or 2 stars in comparability, and 2 or 3 stars in outcomes. A "poor" quality score reflected 0 or 1 star(s) in selection, or 0 stars in comparability, or 0 or 1 star(s) in outcomes.

Statistical analysis was performed using metapackage for $\mathrm{R}$ version 4.0 and Rstudio version 1.2. Mantel-Haenszel risk ratio (RR) random-effects model was used to summarize data between two groups. Heterogeneity of the effect size among the included studies was assessed by Higgins I-squared $\left(I^{2}\right)$ statistic. A value of $I^{2}$ of $0-25 \%$ represented insignificant heterogeneity, $26-50 \%$ represented low heterogeneity, $51-75 \%$ represented moderate heterogeneity, and more than $75 \%$ represented high 


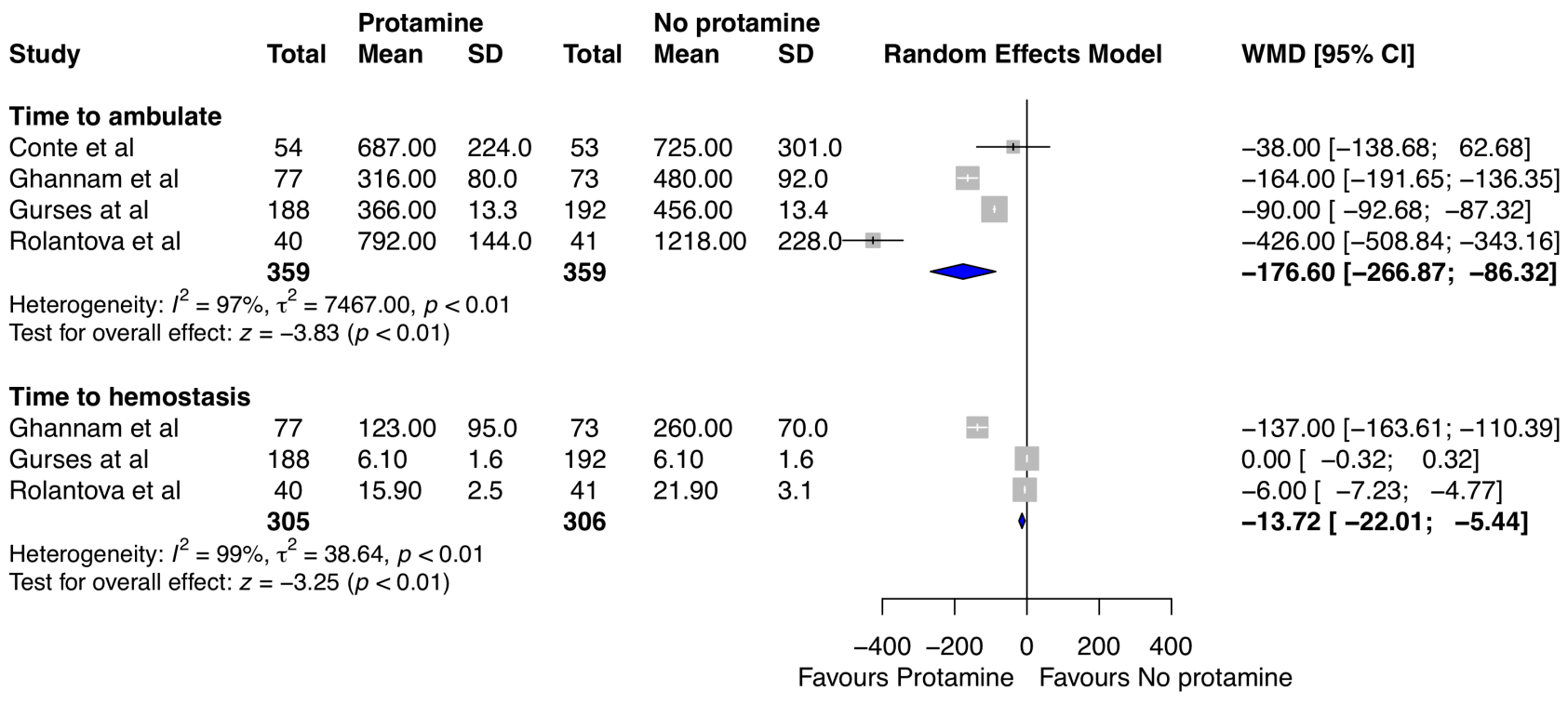

Fig. 2. Forest plot demonstrating risk ratio of adverse events post-procedure between patients who received protamine and patients who did not. The total number of participants and number of events for any vascular complications, minor hematoma, and cerebrovascular accident are reported for each study individually. Square with horizontal line represents RR and 95\% CI for each individual study with square size reflecting the statistical weight of the study using the random-effects model. Diamond demonstrates pooled RR and 95\% CI for each outcome. Heterogeneity $\left(I^{2}\right)$ and between-study variance $\left(T^{2}\right)$ with $p$-value, and overall effect size $(z)$ with $p$-value are reported below each of their respective forest plot. CI, Confidence interval; RR, Risk ratio.

heterogeneity, as set forth by the Cochrane Collaboration. Publication bias was not assessed when the number of studies was less than 10 .

The primary outcomes included - time to hemostasis (minutes) and time to ambulation (minutes). The secondary outcomes included - any vascular complications (composite of any major hematoma, hematomas requiring blood transfusion, pseudoaneurysm, arteriovenous fistula excluding minor hematoma), minor hematoma, or cerebrovascular accidents (CVA).

\section{Results}

A total of 5 eligible studies (3 retrospective cohort studies and two randomized trials) consisting of 1012 patients (515 patients received protamine group and 497 patients did not receive protamine group) were included in the meta-analysis [2-6]. Overall, the mean age was 62.9 \pm 15.6 years, the mean left ventricular ejection fraction was $64.1 \pm 5.9 \%$, and $56.8 \%(\mathrm{n}=575)$ patients were men. About $67.9 \%(\mathrm{n}=687)$ patients had paroxysmal AF, $31 \%(n=314)$ persistent $A F$, and $1.1 \%(n=11)$ were unknown AF duration. Pre-ablation anticoagulation strategies differed amongst studies - from uninterrupted warfarin or minimally interrupted direct oral anticoagulants $[4,6]$ to discontinuing oral anticoagulants or warfarin at least 48 hours before and bridging with low molecular weight heparin if indicated $[3,5]$. 54.25\% $(\mathrm{n}=549)$ patients underwent cryoablation, while $45.75 \%(n=463)$ underwent radiofrequency ablation. The ablation strategy included pul- monary vein isolation and additional ablation at the operator's discretion. In the protamine group, protamine was administered at the end of the procedure with a dose of 1 mg per $100 \mathrm{U}$ of heparin given over 5 minutes, or dosage at the operator's discretion. The sheaths were removed after protamine administration in the protamine group; in contrast, in the no-protamine group, the activated clotting time (ACT) must fall below a target range or after a certain prespecified time period per study protocol. Manual compression and bed rest protocol was implemented in both groups in all studies (Table 1 (Ref. [2-6]) highlights the details on post-procedure management). The two groups were well balanced with respect to baseline demographics and clinical characteristics ( $p$-value $>0.05$ for all). Baseline characteristics of the study population have been detailed in the Table 1.

There was a significant reduction in time to ambulation [weighted mean difference (WMD) -176.6 minutes, $95 \% \mathrm{CI}-266.9$ to $-86.3 ; p<0.01]$ and time to hemostasis (WMD -13.72 minutes, 95\% CI -22 to $-5.4, p<$ $0.01)$ in the protamine group compared to the contrary (Fig. 1). Test of heterogeneity was significant $\left(I^{2} 97 \%\right.$ and $99 \%$, respectively) - primarily due to differences in postprocedure protocols across studies. At a follow-up up to 3 months, there was no statistical difference between the two groups with regards to vascular complications, minor hematoma or CVA. However, protamine group had a numerically lower rates of any vascular complications $(2.9 \%$ vs. $7.4 \%$; RR $0.46,95 \%$ CI 0.17 to 1.24 ; $p=0.12$ ), mi- 


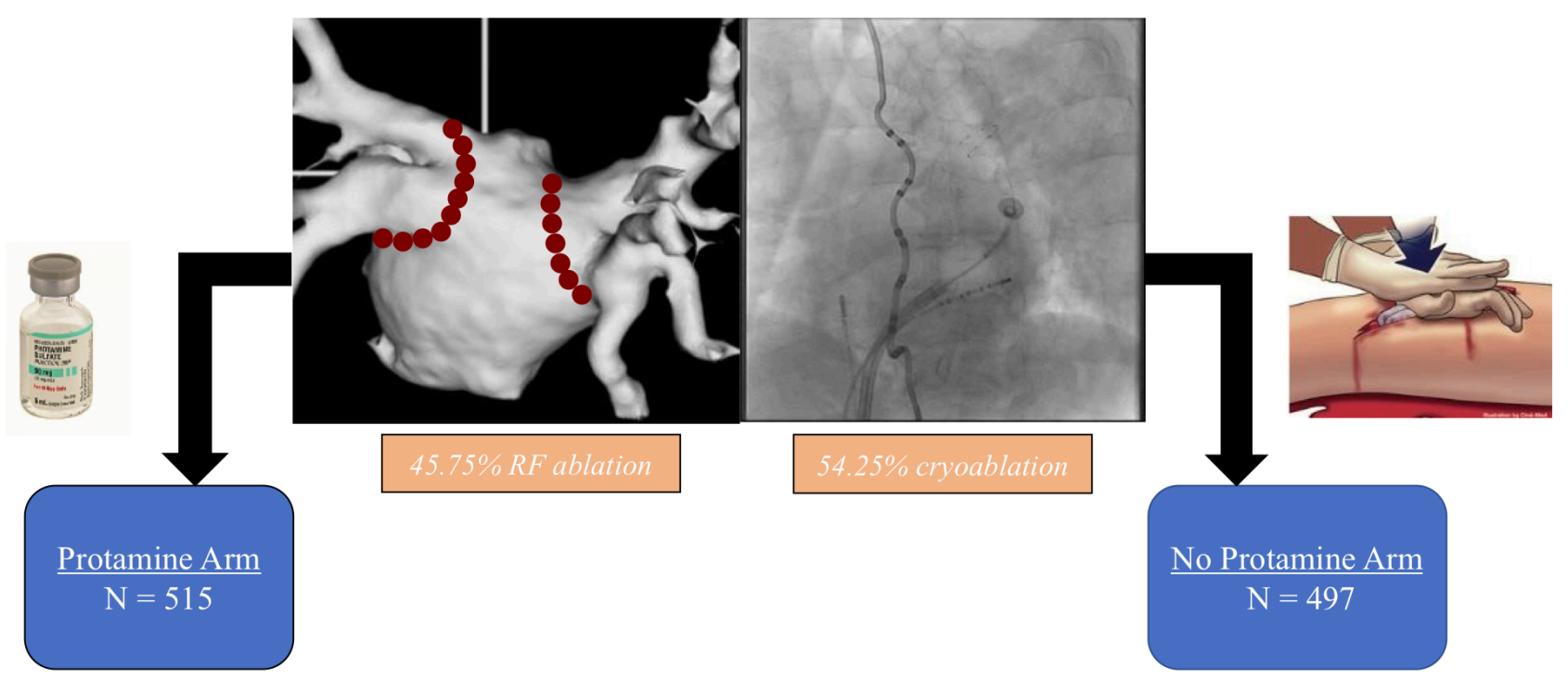

Mean age 62.9 \pm 15.6 years, Mean ejection fraction $=64.1 \pm 5.9 \%$ Paroxysmal Atrial Fibrillation $=\mathbf{6 7 . 9 \%}$, Persistent Atrial fibrillation $=\mathbf{3 1} \%$

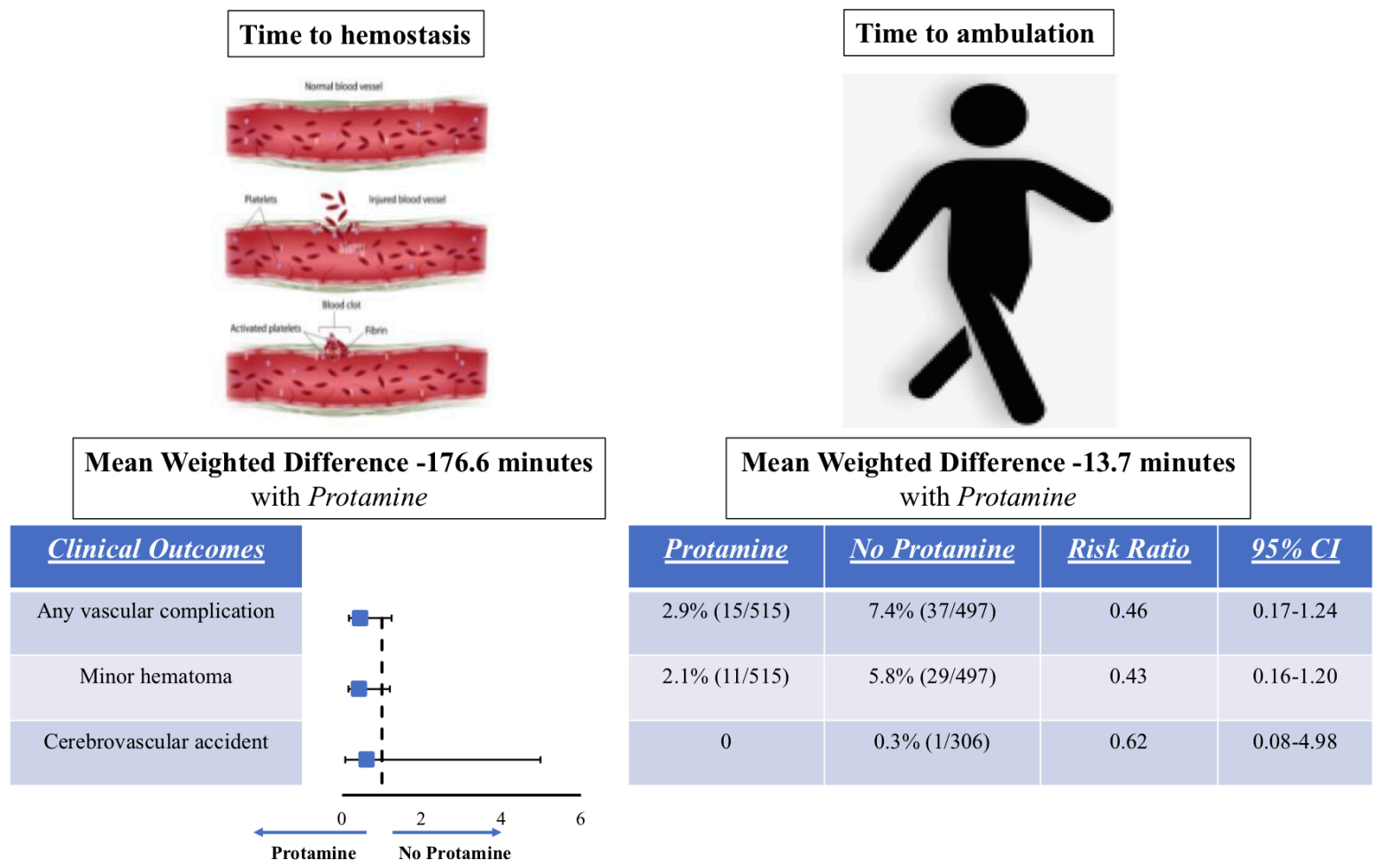

Fig. 3. Protamine administration after catheter ablation of atrial fibrillation.

nor hematoma ( $2.1 \%$ vs. $5.8 \%$; RR $0.43,95 \%$ CI 0.16 to $1.2 ; p=0.11)$ and CVA (0 vs. $0.3 \%$; RR $0.62,95 \%$ CI 0.08 to $4.98 ; p=0.65$ ) when compared with no-protamine group, but the difference did not reach statistical significance (Fig. 2). Reaction to protamine occurred in $3(0.6 \%)$ patients - two patients had hypotension that resolved with fluid administration, and the authors did not define one patient's symptoms.

\section{Discussion}

The results of our pooled analysis demonstrate a significant reduction in time to hemostasis and time to ambulation with a mean of 13 minutes and 176 minutes, respectively, with the use of protamine following AF ablation. There was a positive trend in decreasing the risk of vascular complications and CVA with protamine administration, although this difference did not reach statistical significance due to inadequate power and low event rates (Fig. 3). 
Table 1. Baseline characteristics of the included studies.

\begin{tabular}{|c|c|c|c|c|c|c|c|c|c|c|}
\hline \multirow{2}{*}{ Variables } & \multicolumn{10}{|c|}{ Conte et al. [2], $2014(\mathrm{n}=107)$ Ghannam et al. [4], $2018(\mathrm{n}=150)$ Gurses et al. [3], $2015(\mathrm{n}=380)$ Rolantova et al. [5], 2018 $(\mathrm{n}=81)$ Yasar et al. [6], 2019 $(\mathrm{n}=294)$} \\
\hline & $\begin{array}{l}\text { Protamine } \\
(\mathrm{n}=54)\end{array}$ & $\begin{array}{l}\text { No protamine } \\
\qquad(\mathrm{n}=53)\end{array}$ & $\begin{array}{l}\text { Protamine } \\
(\mathrm{n}=77)\end{array}$ & $\begin{array}{l}\text { No protamine } \\
\quad(\mathrm{n}=73)\end{array}$ & $\begin{array}{l}\text { Protamine } \\
(\mathrm{n}=188)\end{array}$ & $\begin{array}{l}\text { No protamine } \\
\quad(\mathrm{n}=192)\end{array}$ & $\begin{array}{l}\text { Protamine } \\
(\mathrm{n}=40)\end{array}$ & $\begin{array}{l}\text { No protamine } \\
\quad(\mathrm{n}=41)\end{array}$ & $\begin{array}{l}\text { Protamine } \\
(\mathrm{n}=156)\end{array}$ & $\begin{array}{l}\text { No protamine } \\
\quad(\mathrm{n}=138)\end{array}$ \\
\hline Study design & \multicolumn{2}{|c|}{ Retrospective cohort } & \multicolumn{2}{|c|}{ Randomized controlled trial } & \multicolumn{2}{|c|}{ Retrospective cohort } & \multicolumn{2}{|c|}{ Randomized controlled trial } & \multicolumn{2}{|c|}{ Multicenter retrospective cohort } \\
\hline Age, years (mean $\pm \mathrm{SD}$ or median $(\mathrm{SD}))$ & $60 \pm 11$ & $58 \pm 14$ & $63 \pm 12$ & $66 \pm 9$ & $55(27-76)$ & $57(20-86)$ & $61.2 \pm 5.6$ & $64.5 \pm 6.2$ & $64.2 \pm 11.4$ & $63 \pm 11.1$ \\
\hline Male, n (\%) & $33(61 \%)$ & $31(58 \%)$ & $46(60 \%)$ & $48(66 \%)$ & $95(50.5 \%)$ & $88(45.8 \%)$ & $23(58 \%)$ & $22(54 \%)$ & $100(64.1 \%)$ & $89(64.5 \%)$ \\
\hline Diabetes mellitus, n (\%) & $4(7 \%)$ & $5(9 \%)$ & $\mathrm{n} / \mathrm{a}$ & $\mathrm{n} / \mathrm{a}$ & $31(16.5 \%)$ & $25(13 \%)$ & $5(13 \%)$ & $7(17 \%)$ & $24(19.8 \%)$ & $32(23.2 \%)$ \\
\hline Hypertension, n (\%) & $21(39 \%)$ & $23(43 \%)$ & $\mathrm{n} / \mathrm{a}$ & $\mathrm{n} / \mathrm{a}$ & $81(43.1 \%)$ & $94(49 \%)$ & $25(63 \%)$ & $33(81 \%)$ & $91(75.2 \%)$ & $106(76.8 \%)$ \\
\hline Coronary artery disease, $\mathrm{n}(\%)$ & $7(13 \%)$ & $6(11 \%)$ & $\mathrm{n} / \mathrm{a}$ & $\mathrm{n} / \mathrm{a}$ & $17(9 \%)$ & $29(15.1 \%)$ & $5(13 \%)$ & $5(13 \%)$ & $33(27.3 \%)$ & $27(19.6 \%)$ \\
\hline \multicolumn{11}{|l|}{ Type of atrial fibrillation } \\
\hline Paroxysmal, n (\%) & $100 \%$ & $100 \%$ & $40(52 \%)$ & $34(46 \%)$ & $146(88 \%)$ & $163(85 \%)$ & $28(70 \%)$ & $23(56 \%)$ & $92(59 \%)$ & $54(39 \%)$ \\
\hline Persistent, n (\%) & - & - & $34(44 \%)$ & $31(42 \%)$ & $42(22 \%)$ & $29(15 \%)$ & $12(30 \%)$ & $18(44 \%)$ & $64(41 \%)$ & $84(61 \%)$ \\
\hline Anticoagulation use & & & & & $52(27.7 \%)$ & $60(31.3 \%)$ & & & & \\
\hline Warfarin & $31(57 \%)$ & $32(60 \%)$ & $14(18 \%)$ & $14(19 \%)$ & $\mathrm{n} / \mathrm{a}$ & $\mathrm{n} / \mathrm{a}$ & $40(100 \%)$ & $41(100 \%)$ & $22(14.1 \%)$ & $30(21.7 \%)$ \\
\hline NOAC & $9(17 \%)$ & $9(17 \%)$ & $63(82 \%)$ & $59(81 \%)$ & $\mathrm{n} / \mathrm{a}$ & $\mathrm{n} / \mathrm{a}$ & & & $116(74.4 \%)$ & $95(68.8 \%)$ \\
\hline Aspirin & $14(26 \%)$ & $12(23 \%)$ & - & - & $\mathrm{n} / \mathrm{a}$ & $\mathrm{n} / \mathrm{a}$ & & & $\mathrm{n} / \mathrm{a}$ & $\mathrm{n} / \mathrm{a}$ \\
\hline LVEF (mean \pm SD) & $58 \pm 7$ & $55 \pm 8$ & $\mathrm{n} / \mathrm{a}$ & $\mathrm{n} / \mathrm{a}$ & $65.9 \pm 3.1$ & $65.9 \pm 3.5$ & $66.3 \pm 5.2$ & $66 \pm 4.9$ & $\mathrm{n} / \mathrm{a}$ & $\mathrm{n} / \mathrm{a}$ \\
\hline LA size, $\mathrm{mm}($ mean $\pm \mathrm{SD})$ & $41 \pm 6$ & $42 \pm 4$ & $\mathrm{n} / \mathrm{a}$ & $\mathrm{n} / \mathrm{a}$ & $\mathrm{n} / \mathrm{a}$ & $\mathrm{n} / \mathrm{a}$ & $\mathrm{n} / \mathrm{a}$ & $\mathrm{n} / \mathrm{a}$ & $\mathrm{n} / \mathrm{a}$ & $\mathrm{n} / \mathrm{a}$ \\
\hline CHA2DS2-VASc score (mean \pm SD) & $1.8 \pm 1.5$ & $1.6 \pm 1.4$ & $2.1 \pm 1.2$ & $2.2 \pm 1.2$ & $\mathrm{n} / \mathrm{a}$ & $\mathrm{n} / \mathrm{a}$ & $2.2 \pm 1.3$ & $2.1 \pm 1.1$ & $\mathrm{n} / \mathrm{a}$ & $\mathrm{n} / \mathrm{a}$ \\
\hline HAS-BLED score (mean \pm SD) & $1.0 \pm 1.9$ & $0.9 \pm 0.9$ & $\mathrm{n} / \mathrm{a}$ & $\mathrm{n} / \mathrm{a}$ & $\mathrm{n} / \mathrm{a}$ & $\mathrm{n} / \mathrm{a}$ & $\mathrm{n} / \mathrm{a}$ & $\mathrm{n} / \mathrm{a}$ & $\mathrm{n} / \mathrm{a}$ & $\mathrm{n} / \mathrm{a}$ \\
\hline Pre-ablation anticoagulation strategies & 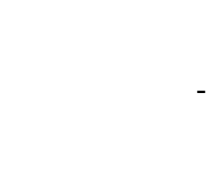 & & \multicolumn{2}{|c|}{$\begin{array}{l}\text { uninterrupted warfarin or } \\
\text { minimally interrupted } \\
\text { oral anticoagulants }\end{array}$} & \multicolumn{4}{|c|}{$\begin{array}{cc}\text { discontinuing oral anticoagulants discontinuing oral anticoagulants } \\
\text { or warfarin } 48 \text { hours before; } & \text { or warfarin } 48 \text { hours before; } \\
\text { bridging with low molecular } & \text { bridging with low molecular } \\
\text { weight heparin } & \text { weight heparin }\end{array}$} & \multicolumn{2}{|c|}{$\begin{array}{l}\text { uninterrupted warfarin or } \\
\text { minimally interrupted } \\
\text { oral anticoagulants }\end{array}$} \\
\hline \multicolumn{11}{|l|}{ Ablation type } \\
\hline $\begin{array}{l}\text { Radiofrequency, } \mathrm{n} \\
(\%)\end{array}$ & - & - & $57(74 \%)$ & $55(75 \%)$ & - & - & $100 \%$ & $100 \%$ & $132(85 \%)$ & $100 \%$ \\
\hline Cryoballoon, n (\%) & $100 \%$ & $100 \%$ & $20(26 \%)$ & $18(25 \%)$ & $100 \%$ & $100 \%$ & - & - & $24(15 \%)$ & - \\
\hline Total heparin dose, $U$ (mean \pm SD) & $8900 \pm 2500$ & $8700 \pm 2600$ & $23724 \pm 13977$ & $21541 \pm 13649$ & $7808 \pm 1281$ & $7827 \pm 1270$ & $19500 \pm 2700$ & $18900 \pm 3200$ & $\begin{array}{c}239 \pm 69 \\
(\mathrm{U} / \mathrm{kg})\end{array}$ & $\begin{array}{c}208 \pm 56 \\
(\mathrm{U} / \mathrm{kg})\end{array}$ \\
\hline Protamine administration & $\begin{array}{l}1 \mathrm{mg} / 100 \mathrm{U} \text { of } \\
\text { heparin given } \\
\text { at the end of } \\
\text { procedure }\end{array}$ & - & $\begin{array}{l}1 \mathrm{mg} / 100 \mathrm{U} \text { of } \\
\text { heparin given at } \\
\text { the end of } \\
\text { procedure }\end{array}$ & - & $\begin{array}{l}1 \mathrm{mg} / 100 \mathrm{U} \text { of } \\
\text { heparin given } \\
\text { at the end of } \\
\text { procedure }\end{array}$ & - & $\begin{array}{l}\text { Fixed dose per } \\
\text { total heparin } \\
\text { and last ACT } \\
\text { given at the end } \\
\text { of procedure }\end{array}$ & - & $\begin{array}{l}\text { Dose at } \\
\text { operator's } \\
\text { discretion }\end{array}$ & - \\
\hline
\end{tabular}


Table 1. Continued.

\begin{tabular}{|c|c|c|c|c|c|c|c|c|c|c|}
\hline \multirow{2}{*}{ Variables } & \multicolumn{8}{|c|}{ Conte et al. [2], $2014(\mathrm{n}=107)$ Ghannam et al. [4], $2018(\mathrm{n}=150)$ Gurses et al. [3], $2015(\mathrm{n}=380)$ Rolantova et al. [5], $2018(\mathrm{n}=81)$} & \multicolumn{2}{|c|}{ Yasar et al. [6], $2019(\mathrm{n}=294)$} \\
\hline & $\begin{array}{l}\text { Protamine } \\
(\mathrm{n}=54)\end{array}$ & $\begin{array}{l}\text { No protamine } \\
\quad(\mathrm{n}=53)\end{array}$ & $\begin{array}{l}\text { Protamine } \\
(\mathrm{n}=77)\end{array}$ & $\begin{array}{l}\text { No protamine } \\
\quad(\mathrm{n}=73)\end{array}$ & $\begin{array}{l}\text { Protamine } \\
(\mathrm{n}=188)\end{array}$ & $\begin{array}{l}\text { No protamine } \\
\quad(\mathrm{n}=192)\end{array}$ & $\begin{array}{l}\text { Protamine } \\
(\mathrm{n}=40)\end{array}$ & $\begin{array}{l}\text { No protamine } \\
\quad(\mathrm{n}=41)\end{array}$ & $\begin{array}{l}\text { Protamine } \\
(\mathrm{n}=156)\end{array}$ & $\begin{array}{l}\text { No protamine } \\
\quad(\mathrm{n}=138)\end{array}$ \\
\hline Sheath removal process & $\begin{array}{l}\text { At the end of } \\
\text { protamine } \\
\text { administration }\end{array}$ & $\begin{array}{l}\text { After ACT } \\
\quad<150\end{array}$ & \multicolumn{2}{|c|}{$\begin{array}{c}\text { After ACT }<200 \text { or } \\
\text { pre-procedural baseline }\end{array}$} & $\begin{array}{l}\text { At the end of } \\
\text { protamine } \\
\text { administration }\end{array}$ & $\begin{array}{l}90 \text { minutes } \\
\text { after last } \\
\text { heparin dose } \\
\text { without ACT }\end{array}$ & $\begin{array}{l}\text { At the end of } \\
\text { protamine } \\
\text { administration }\end{array}$ & $\begin{array}{c}\text { After ACT } \\
<1.5 \text { times } \\
\text { upper normal } \\
\text { limit }\end{array}$ & $\begin{array}{l}\text { After ACT }<200 \\
\text { or at the end of } \\
\text { protamine } \\
\text { administration }\end{array}$ & $\begin{array}{l}\text { Suture with } \\
\text { F8S or SCT } \\
\text { prior to sheath } \\
\text { removal }\end{array}$ \\
\hline Hemostasis-to-ambulation protocol & \multicolumn{2}{|c|}{$\begin{array}{c}\text { Manual compression and } \\
\text { 10-hr bed rest and } \\
\text { groin bandage }\end{array}$} & \multicolumn{2}{|c|}{$\begin{array}{l}\text { Manual compression and } \\
\text { 4-hr bed rest }\end{array}$} & \multicolumn{2}{|c|}{$\begin{array}{c}\text { Manual compression and 6-hr } \\
\text { bed rest and 12-hr } \\
\text { groin bandage }\end{array}$} & \multicolumn{2}{|c|}{$\begin{array}{l}\text { Manual compression and } \\
\text { 12-hr bed rest }\end{array}$} & \multicolumn{2}{|c|}{$\begin{array}{l}\text { Manual compression and } \\
\text { 4-hr bedrest for both group }\end{array}$} \\
\hline \multicolumn{11}{|l|}{ Procedural time (minutes) } \\
\hline Total time $($ mean $\pm \mathrm{SD})$ & $93 \pm 8$ & $89 \pm 12$ & $199 \pm 74$ & $214 \pm 40$ & $73.6 \pm 12.6$ & $71.3 \pm 12.1$ & $111 \pm 13$ & $104 \pm 14$ & $177.1 \pm 48.1$ & $213.2 \pm 57.3$ \\
\hline $\begin{array}{l}\text { Fluoroscopy time } \\
(\text { mean } \pm \mathrm{SD})\end{array}$ & $14 \pm 6$ & $13 \pm 6$ & $\mathrm{n} / \mathrm{a}$ & $\mathrm{n} / \mathrm{a}$ & $15 \pm 2.5$ & $15.1 \pm 2.7$ & $4.8 \pm 1.1$ & $5.1 \pm 1.3$ & $\mathrm{n} / \mathrm{a}$ & $\mathrm{n} / \mathrm{a}$ \\
\hline \multicolumn{11}{|l|}{ Post-procedural time (minutes) } \\
\hline $\begin{array}{l}\text { Time to sheath removal } \\
(\text { mean } \pm \mathrm{SD})\end{array}$ & $\mathrm{n} / \mathrm{a}$ & $\mathrm{n} / \mathrm{a}$ & $\mathrm{n} / \mathrm{a}$ & $\mathrm{n} / \mathrm{a}$ & $6.3 \pm 2.4$ & $96.3 \pm 2.4$ & $\mathrm{n} / \mathrm{a}$ & $\mathrm{n} / \mathrm{a}$ & $\mathrm{n} / \mathrm{a}$ & $\mathrm{n} / \mathrm{a}$ \\
\hline $\begin{array}{l}\text { Time to hemostasis } \\
\text { (mean } \pm \mathrm{SD})\end{array}$ & $\mathrm{n} / \mathrm{a}$ & $\mathrm{n} / \mathrm{a}$ & $123 \pm 95$ & $260 \pm 70$ & $6.1 \pm 1.6$ & $6.1 \pm 1.6$ & $15.9 \pm 2.5$ & $21.9 \pm 3.1$ & $\mathrm{n} / \mathrm{a}$ & $\mathrm{n} / \mathrm{a}$ \\
\hline $\begin{array}{l}\text { Time to ambulation } \\
\text { (mean } \pm \mathrm{SD})\end{array}$ & $687 \pm 224$ & $725 \pm 301$ & $316 \pm 80$ & $480 \pm 92$ & $366.3 \pm 13.3$ & $456.3 \pm 13.4$ & $792 \pm 144$ & $1218 \pm 228$ & $\mathrm{n} / \mathrm{a}$ & $\mathrm{n} / \mathrm{a}$ \\
\hline Reported vascular complications & \multicolumn{2}{|c|}{ Hematoma, bleeding } & \multicolumn{2}{|c|}{ Bleeding, hematoma } & \multicolumn{2}{|c|}{$\begin{array}{c}\text { Hematoma, pseudoaneurysm, } \\
\text { AVF }\end{array}$} & \multicolumn{2}{|c|}{$\begin{array}{l}\text { Hematoma, pseudoaneurysm, } \\
\text { AVF, bleeding }\end{array}$} & \multicolumn{2}{|c|}{$\begin{array}{l}\text { Hematoma, pseudoaneurysm, } \\
\text { AVF, suture failure }\end{array}$} \\
\hline \multicolumn{11}{|l|}{ Adverse events } \\
\hline $\begin{array}{l}\text { Any Vascular compli- } \\
\text { cation, } \mathrm{n}(\%)\end{array}$ & 0 & $6(11 \%)$ & $6(8 \%)$ & $4(5 \%)$ & $2(1.1 \%)$ & $12(6.3 \%)$ & $5(12.5 \%)$ & $14(34 \%)$ & $2(1 \%)$ & $1(0.6 \%)$ \\
\hline Minor hematoma, n (\%) & 0 & $5(9 \%)$ & $5(6 \%)$ & $4(5 \%)$ & $1(0.5 \%)$ & $8(4 \%)$ & $4(10 \%)$ & $12(29.2 \%)$ & $1(0.6 \%)$ & 0 \\
\hline $\begin{array}{l}\text { Cerebrovascular acci- } \\
\text { dent, } \mathrm{n}(\%)\end{array}$ & $\mathrm{n} / \mathrm{a}$ & $\mathrm{n} / \mathrm{a}$ & $0(0 \%)$ & $1(1 \%)$ & $0(0 \%)$ & $0(0 \%)$ & $0(0 \%)$ & $0(0 \%)$ & $\mathrm{n} / \mathrm{a}$ & $\mathrm{n} / \mathrm{a}$ \\
\hline $\begin{array}{l}\text { Adverse reaction to } \\
\text { protamine }\end{array}$ & 0 & - & $1(1 \%)$ & - & $1(0.5 \%)$ & - & 0 & - & $1(0.6 \%)$ & - \\
\hline
\end{tabular}


Vascular access complications, including bleeding, hematoma, arteriovenous fistula, or pseudoaneurysm, are major adverse events in patients undergoing AF ablation due to an aggressive anticoagulation protocol [7-9]. To reduce the risk of bleeding events, ACT must return from ACT goal during the procedure (300-350 s) to normal range $(<180 \mathrm{~s})$ before femoral sheath removal [1]. However, this process can take up to several hours due to a 90-min halflife of UFH, thus creating a negative experience for the patients. Furthermore, the risk of vascular complications increases the longer the sheath remains in the femoral vein [10]. Protamine sulfate binds with heparin to form inactive complexes and rapidly negates the antithrombotic effect of heparin [11]. Despite the long history of its utilization in other specialties, including cardiothoracic surgery and interventional cardiology $[12,13]$, the evidence of its safety and efficacy in AF ablation is limited. This is the first systematic review and meta-analysis to evaluate the effectiveness and safety of protamine in this patient population.

Taken together, our study provides the best available evidence to date regarding the effect of protamine administration on sheath removal time, immobilization time, and the risk of adverse clinical events - finding which might be clinically relevant and can reduce intensive care monitoring time, and consequently health care cost utilization. This meta-analysis is limited by possible patient-physician selection bias, lack of patient-level data, heterogeneous study design (different preablation anticoagulation management strategies) and follow-up period, and lack of long-term outcomes and imaging data. Finally, these results cannot be extrapolated to groins closed with commercially available vascular closure devices.

\section{Author contributions}

Conceptualization: JK, KS, MT; methodology, JK, KS, MT, JG; software, JK, KS; validation, KS, MT, JG; formal analysis, RB, RM, TC; investigation, MT, RB, JG; resources, $\mathrm{KS}$, JG; data curation, JK, KS; writing — original draft preparation, JK, TC, DL; writing - review and editing, DL, JG; visualization, RB, TC, RM; supervision, DL, JG; project administration RM, DL, JG. All authors have read and agreed to the published version of the manuscript.

\section{Ethics approval and consent to participate}

Not applicable.

\section{Acknowledgment}

We would like to express our gratitude to all those who helped us during the writing of this manuscript. Thanks to all the peer reviewers for their opinions and suggestions.

\section{Funding}

This research received no external funding.

\section{Conflict of interest}

The authors declare no conflict of interest.

\section{Supplementary material}

Supplementary material associated with this article can be found, in the online version, at https://www.imrpre ss.com/journal/RCM/23/1/10.31083/j.rcm2301034.

\section{References}

[1] Calkins H, Hindricks G, Cappato R, et al. 2017 HRS/EHRA/ECAS/APHRS/SOLAECE expert consensus statement on catheter and surgical ablation of atrial fibrillation. Europace. 2018; 20: e1-e160.

[2] Conte G, de Asmundis C, Baltogiannis G, Di Giovanni G, Ciconte G, Sieira J, et al. Periprocedural outcomes of prophylactic protamine administration for reversal of heparin after cryoballoon ablation of atrial fibrillation. Journal of Interventional Cardiac Electrophysiology. 2014; 41: 129-134.

[3] Gurses KM, Kocyigit D, Yalcin MU, Evranos B, Yorgun H, Sahiner ML, et al. Safety and efficacy outcomes of protamine administration for heparin reversal following cryoballoon-based pulmonary vein isolation. Journal of Interventional Cardiac Electrophysiology. 2015; 43: 161-167.

[4] Ghannam M, Chugh A, Dillon P, Alyesh D, Kossidas K, Sharma $\mathrm{S}$, et al. Protamine to expedite vascular hemostasis after catheter ablation of atrial fibrillation: a randomized controlled trial. Heart Rhythm. 2018; 15: 1642-1647.

[5] Rolantova L, Bulava A, Eisenberger M, Chloubova I, Tothova V, Hanis J. Nurse-performed venous sheath removal in patients undergoing radiofrequency catheter ablation for atrial fibrillation: a randomised study. European Journal of Cardiovascular Nursing. 2019; 18: 332-339.

[6] Yasar SJ, Bickel T, Zhang S, Akkaya M, Aznaurov SG, Krishnan $\mathrm{K}$, et al. Heparin reversal with protamine sulfate is not required in atrial fibrillation ablation with suture hemostasis. Journal of Cardiovascular Electrophysiology. 2019; 30: 2811-2817.

[7] Tripathi B, Arora S, Kumar V, Abdelrahman M, Lahewala S, Dave M, et al. Temporal trends of in-hospital complications associated with catheter ablation of atrial fibrillation in the United States: an update from Nationwide Inpatient Sample database (2011-2014). Journal of Cardiovascular Electrophysiology. 2018; 29: 715-724.

[8] Yang E, Ipek EG, Balouch M, Mints Y, Chrispin J, Marine JE, et al. Factors impacting complication rates for catheter ablation of atrial fibrillation from 2003 to 2015. Europace. 2017; 19: 241249.

[9] Hoyt H, Bhonsale A, Chilukuri K, Alhumaid F, Needleman M, Edwards D, et al. Complications arising from catheter ablation of atrial fibrillation: temporal trends and predictors. Heart Rhythm. 2011; 8: 1869-1874.

[10] Spragg DD, Dalal D, Cheema A, Scherr D, Chilukuri K, Cheng A, et al. Complications of catheter ablation for atrial fibrillation: incidence and predictors. Journal of Cardiovascular Electrophysiology. 2008; 19: 627-631.

[11] Sokolowska E, Kalaska B, Miklosz J, Mogielnicki A. The toxicology of heparin reversal with protamine: past, present and future. Expert Opinion on Drug Metabolism and Toxicology. 2016; 12: 897-909.

[12] Boer C, Meesters MI, Veerhoek D, Vonk ABA. Anticoagulant and side-effects of protamine in cardiac surgery: a narrative review. British Journal of Anaesthesia. 2018; 120: 914-927.

[13] De Luca G, Parodi G, Antoniucci D. Safety and benefits of protamine administration to revert anticoagulation soon after coronary angioplasty. A meta-analysis. Journal of Thrombosis and Thrombolysis. 2010; 30: 452-458. 